Questions vives

\section{Questions Vives}

Recherches en éducation

$N^{\circ} 31 \mid 2019$

Évaluation et numérique : Nouvelles pratiques ?

Nouvelles opportunités?

\title{
Naissance d'une coopération dans un ESAT : quand des moniteurs d'atelier, un travailleur et un chercheur apprennent à travailler ensemble
}

Birth of cooperation in an ESAT: when workshop instructors, a worker and a researcher learn to work together.

\section{Caroline Perraud}

\section{(2) OpenEdition}

\section{Journals}

\section{Édition électronique}

URL : http://journals.openedition.org/questionsvives/4011

DOI : 10.4000/questionsvives.4011

ISSN : 1775-433X

Éditeur

Université Aix-Marseille (AMU)

\section{Édition imprimée}

Date de publication : 30 juillet 2019

ISBN : 978-2-912643-55-1

ISSN : 1635-4079

\section{Référence électronique}

Caroline Perraud, « Naissance d'une coopération dans un ESAT : quand des moniteurs d'atelier, un travailleur et un chercheur apprennent à travailler ensemble », Questions Vives [En ligne], № 31 | 2019, mis en ligne le 30 août 2020, consulté le 03 avril 2021. URL : http://journals.openedition.org/ questionsvives/4011; DOI : https://doi.org/10.4000/questionsvives.4011

Ce document a été généré automatiquement le 3 avril 2021.

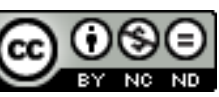

Questions Vives est mis à disposition selon les termes de la licence Creative Commons Attribution -

Pas d'Utilisation Commerciale - Pas de Modification 4.0 International. 


\section{Naissance d'une coopération dans un ESAT : quand des moniteurs d'atelier, un travailleur et un chercheur apprennent à travailler ensemble}

Birth of cooperation in an ESAT: when workshop instructors, a worker and a researcher learn to work together.

\section{Caroline Perraud}

\section{Introduction}

1 La visée de cet article est de décrire et analyser les premiers pas d'une coopération dans un établissement et service d'aide par le travail (ESAT), entre des professionnels, des adultes en situation de handicap (appelés travailleurs) et une chercheure, anciennement enseignante spécialisée (l'auteure de ces lignes). Ensemble et sous l'impulsion de l'Apei Ouest $44^{1}$, ce collectif a appris à travailler ensemble à la construction et à la mise en œuvre de situations d'enseignement-apprentissage pour les travailleurs. Plus spécifiquement, ce collectif s'est interrogé sur la place accordée aux savoirs fondamentaux (Lire, écrire et compter) pour les adultes de ces établissements et services. Ce collectif a progressivement pris la forme d'une ingénierie coopérative, qui sera détaillée dans la partie " éléments de théorie ».

2 Dans cet article, nous proposons de répondre à la question suivante : que se passe-t-il quand des acteurs de cultures différentes apprennent à travailler ensemble? Qu'est-ce que cette coopération inédite transforme?

3 Nous nous centrerons sur la coopération dans l'atelier restauration de l'ESAT, entre un travailleur, Walter, les monitrices de cet atelier et la chercheure. Ensemble, ils vont 
résoudre un problème ${ }^{2}$ issu de la pratique de l'activité de Walter dans l'atelier restauration. Nous examinerons la genèse d'un outil d'accessibilité ${ }^{3}$, appelé cartesconstellations, vues comme objet-solution (Baxandall, 1991). En tant que pratique inédite au début du projet, la production de ces cartes-constellations constitue à notre sens un exemple emblématique $^{4}$ (Sensevy, 2011; Joffredo-Lebrun 2016 ; Morellato, 2017) de la naissance de la coopération dans les ateliers. Après une présentation de notre étude, nous décrirons, puis analyserons la genèse de ces cartes constellations. Nous terminerons par une courte discussion.

\section{Présentation de l'étude}

\section{1. Éléments de théorie}

Notre cadre théorique s'ancre dans la théorie de l'action conjointe en didactique (Sensevy, 2011) qui tente de comprendre l'enseignement et l'apprentissage en contexte.

5 Sensevy (2011) a défini une ingénierie coopérative comme un lieu de partage de pratique. Une ingénierie coopérative « est un art de faire qui s'apprend, tout en étant l'instrument de sa propre institution. Une ingénierie coopérative ne se décrète donc pas, elle se vit » (Perraud, 2018b). Elle regroupe un certain nombre d'acteurs (praticiens et chercheurs) qui apprennent à résoudre ensemble des problèmes, dans un long travail d'enquête collectif en appui sur des principes.

6 Le principe de symétrie dans lequel «chacun a la responsabilité de s'exprimer et doit prendre en compte les expressions des autres » (Perraud, 2018a) est un point de départ à la coopération, en permettant aux différents acteurs de s'enrichir de leurs pratiques respectives, tout en enrichissant les pratiques de chacun et la recherche elle-même. Ainsi, les acteurs d'une ingénierie coopérative travaillent ensemble dans une relation épistémique coopérative (Joffredo-Lebrun et al., 2017) qui tend vers cette symétrie : en fonction du problème travaillé, ceux qui savent quelque chose (des connaisseurs pratiques $^{5}$ ) partagent ce quelque chose avec les autres (des non connaisseurs pratiques). Ce partage permet au collectif de travailler ensemble à la construction de solutions adéquates à la logique de la pratique dans laquelle réside le problème, le problème de la pratique. Le moteur du travail d'enquête collectif est constitué dans cette action conjointe par des dissymétries temporaires et partagées, prenant la forme de controverses d'ingénierie (Perraud, 2018b). Ainsi, une ingénierie coopérative est un espace prenant en compte les différences, qui constitue un autre principe de l'ingénierie coopérative : le principe d'assomption des différences (Joffredo-Lebrun et al., 2017).

7 Le travail d'enquête collectif est centré sur les savoirs, c'est donc une enquête épistémique. Précisons que nous entendons les savoirs comme des puissances d'agir (Sensevy, 2011) car ils permettent de mieux agir dans une situation donnée ${ }^{6}$. Mais, c'est aussi une enquête scientifique. En effet, c'est « une recherche dans laquelle praticiens et chercheurs transforment ensemble des pratiques, et, les transformant, en comprennent mieux les constituants fondamentaux. Chercheurs et praticiens sont autant co-chercheurs que co-praticiens. Ils sont ingénieurs, et un autre principe est ainsi concrétisé : celui de la posture d'ingénieur» (Perraud, 2019). Cette posture confère à chacun la possibilité de participer à l'invention collective de solutions aux problèmes rencontrés et de les expérimenter concrètement (dans la pratique). Une ingénierie coopérative permet donc dans un même temps de comprendre/transformer 
autant les pratiques, les instruments de ces pratiques que les acteurs la constituant. C'est ce que nous nous proposons de déceler dans cet article.

\subsection{La restauration, un atelier d'ESAT}

8 Un ESAT s'appuie sur diverses activités de production d'équipes de travailleurs, encadrés par des moniteurs d'ateliers. Simultanément, ces moniteurs offrent un accompagnement à la personne, comme dans tout établissement du secteur médicosocial. La mission des ESAT est donc duelle. Cette dualité production/ accompagnement est donc à prendre en compte.

Dans cet article, centré sur l'atelier restauration ${ }^{7}$, la " production » passe par un service pour une clientèle. Les moniteurs d'ateliers des ESAT doivent alors veiller à ce que le rythme de production (la préparation et le service des repas) et le rythme du travailleur accompagné soient adéquats.

\section{2. Éléments de méthode}

Notre méthodologie, à la fois ethnographique et clinique (Sensevy, 1999 ; Leutenegger, 2000), se fonde aussi sur une approche collaborative (Desgagné et al., 2001, Daguzon, Marlot et Toullec-Théry, 2017). En tant que chercheure, nous étudions l'ingénierie coopérative mise en place dans l'ESAT, mais nous avons agi également directement dans les ateliers auprès des travailleurs de l'ESAT avec les moniteurs d'atelier, en nous appuyant sur notre pratique antérieure d'enseignante-spécialisée.

$11 \mathrm{Au}$ début du projet, le contexte de l'ESAT est inconnue pour la chercheure. C'est pourquoi nous avons débuté notre étude par un temps d'observations in situ des pratiques des ateliers en ESAT. Nous avons décrit ces pratiques observées in situ dans un journal de terrain. Nous avons ajouté à ces descriptions, un compte-rendu des échanges que nous avons eus avec chacun des professionnels rencontrés.

Après ce travail de prise de contact, quelques professionnels de l'ESAT ont fait le choix de participer au projet. Il a débuté auprès de dix travailleurs d'ESAT volontaires. Ceuxci présentaient des "profils » différents. Par exemple, un travailleur peu à l'aise avec l'écrit, excellait dans la maintenance des tondeuses. Une autre travailleuse lectrice ne prenait aucune initiative sur les chantiers des espaces verts. Le groupe était ainsi constitué de lecteurs ou non lecteurs avec peu ou beaucoup de compétences professionnelles. Dès lors, la visée commune était de s'appuyer sur les compétences des travailleurs pour qu'ils progressent dans des domaines où ils pouvaient être en difficulté.

Dans sa mise en œuvre, le projet s'est donc appuyé sur deux actions complémentaires :

- La chercheure est intervenue directement dans les ateliers de l'ESAT, auprès d'un ou deux travailleurs. Elle a mené des séances co-construites avec le moniteur d'atelier. Les coopérations moniteurs, chercheure et travailleurs. C'est un tel dispositif que nous étudierons dans cet article ;

- Douze réunions de l'ingénierie coopérative ont réuni l'ensemble des professionnels volontaires. Elles ont jalonné le dispositif tout au long de son déploiement.

Précisons que le rythme des interventions de la chercheure auprès des travailleurs a été défini avec les moniteurs. Ainsi, nous nous rendions dans les ateliers, chaque 
semaine, à hauteur de trente à quarante-cinq minutes. Nos observations et analyses étaient consignées dans notre journal de terrain. Nous l'avons complété de photos, prises avec un smartphone, de tout ce qui pouvait enrichir nos descriptions ethnographiques. Puisque nous intervenions auprès des travailleurs, notre posture s'est apparentée à celle de l'observation-participante (Malinowski,1963/1922). S'ajoutent à ce corpus, les transcriptions à partir des enregistrements audios des dialogues de l'ingénierie coopérative.

Dans notre étude, les analyses de données sont menées en produisant un mouvement ascendant de l'abstrait au concret (Ilyenkov, 1982 ; Davydov, 1990 ; Engeström 1990) en tant que possible «modalité de transmission des savoirs» (CDpE, 2019, p. 592) ${ }^{8}$. Dans cet article, nous reprenons le même modus operandi, c'est-à-dire que nous allons décrire, après examen des journaux de terrain, dans un langage commun, la genèse et le rôle de cartes-constellations créées au sein de l'atelier restauration. Puis, nous nous focaliserons sur certains aspects de cette première description, en produisant une seconde description théorique, en appui sur quelques concepts issus de la TACD. Ce second moment qui constitue notre analyse, nous permettra de mettre au jour des aspects de la coopération naissante entre les acteurs (monitrices, chercheure et travailleur).

\section{Un exemple emblématique, les cartes constellations}

\subsection{Un problème dans l'activité de restauration de Walter}

Lors de notre première visite dans l'atelier, Walter occupait ce jour-là le poste "préparation des desserts ». Dans ses observations, la chercheure a décelé plusieurs situations convoquant les nombres. Elles impliquaient tant la lecture de nombre, que la constitution d'une quantité d'objets et la constitution de deux collections équipotentes. Walter a ainsi été amené à dénombrer vingt-sept prunes. Il a également déposé six bananes dans six ramequins. Enfin, un travailleur, collègue de Walter, a préparé vingtcinq petites assiettes à fromage.

Chaque fois que Walter a rencontré de telles situations, ses réussites et les obstacles rencontrés ont été repérés. Ainsi, nous avons observé que si Walter énonce la comptine numérique sans erreur jusqu'à 7 , il ne peut en revanche constituer une collection d'objets puisque 1 / sa connaissance de la comptine numérique est lacunaire et 2/ l'adéquation mot-nombre/geste n'est pas stabilisée (il y a un décalage entre le pointage des objets à dénombrer et l'énonciation de la comptine numérique). Par conséquent, la présence d'une des monitrices d'atelier est nécessaire afin qu'elle accompagne Walter dans l'énonciation des mots-nombres pour dénombrer. Ce constat semble pouvoir offrir un espace à la chercheure.

Pour préparer cette intervention, nous avons procédé à un affinement des observations de Walter, en nous appuyant sur tous les indices récoltés lors de la visite de l'atelier. En particulier, nous avons exploré les rapports que Walter entretient avec les nombres. Ainsi, quand Walter met chacune des six bananes dans chacun des six ramequins, il montre qu'il est capable de faire correspondre terme à terme deux collections distinctes d'éléments, c'est-à-dire d'utiliser le principe de bijection (Gelman \& Gallistel, 1978). De plus, il dispose de bonnes capacités de discrimination visuelle. Un travail sur des configurations canoniques ou ce que Brissiaud (2014) appelle la collection-témoin 
organisée, peut permettre à Walter de constituer une collection d'objets. La vérification de la quantité est alors à deux niveaux :

1. niveau "perception visuo-spatiale »: Walter, en comparant la disposition spatiale d'objets avec une configuration canonique, peut vérifier s'il a correctement quantifié les objets.

2. niveau " comptage »: Warren peut par la suite pointer un à un les objets et percevoir que le dernier mot-nombre énoncé correspondant à la quantité commandée.

Enfin, si la nécessité de production dans le travail apporte des contraintes, ne peut-elle favoriser chez Walter le sens et la compréhension de la numérosité ? En effet, la situation de travail peut lui permettre d'expérimenter le processus de quantification. Quoi qu'il en soit, pour que Walter puisse utiliser, dans son contexte professionnel, des collections-témoins organisées pour constituer des collections d'objets, il apparait pertinent de s'orienter vers la fabrication d'outils d'accessibilité s'appuyant sur ses compétences déjà-là.

20 A l'aune de ces constats et observations, un problème que nous formulons ainsi "permettre à Walter de constituer seul des collections d'objets » émerge. Voyons comment ce problème devient un problème commun au petit collectif (moniteurs, travailleur et chercheur).

\subsection{Amorce d'un travail d'enquête collectif : devenir un problème commun et concevoir ensemble des solutions}

Il se trouve que les monitrices d'atelier constatent elles-aussi que les difficultés de dénombrement chez Walter entravent effectivement son activité professionnelle. Aussi, elles adhèrent à l'idée de pouvoir réfléchir ensemble à des solutions. S'appuyant alors sur son expérience, son étude de la situation, et tenant compte des contraintes de l'atelier, la chercheure suggère le recours à deux outils d'accessibilité au dénombrement: les cartes-constellations et les pastilles. Pourquoi ces deux outils d'accessibilité ont-ils été envisagés? Considérons un problème-type parmi ceux que doit résoudre Walter.

Imaginons que Walter a en charge la préparation de vingt-cinq petites assiettes à fromage. Pour ce faire, il doit d'abord dénombrer ces vingt-cinq petites assiettes. Puis, il disposera dans chacune une portion de fromage, une portion de beurre et une feuille de salade pour la décoration. Comme nous l'avons dit, Walter n'a pas stabilisé sa comptine numérique. Nous pouvons donc aisément envisager que dénombrer vingtcinq assiettes va lui poser problème. Dans ses pratiques antérieures, la chercheure a conçu des outils pour des élèves en situation de handicap présentant les mêmes forces et faiblesses que Walter. Il s'agissait d'utiliser les configurations canoniques des constellations du dé pour compter. Les quantités de 1 à 6 étaient représentées sur des cartes, appelées cartes-constellations (cf. paragraphe suivant). Comment alors reprendre cette idée de l'outil "cartes-constellations" en l'adaptant à l'atelier restauration?

\subsubsection{Les cartes-constellations}

En appui sur son expérience antérieure d'enseignante spécialisée, la chercheure propose aux monitrices d'atelier de fabriquer ces cartes-constellations, où figurent des collections témoins organisées. La monitrice d'atelier présente lors de cet échange 
accueille cette solution comme solution possiblement adéquate à l'activité professionnelle de l'atelier restauration. Sa collègue fera de même par la suite. Chercheure et monitrices sont donc d'accord pour mettre en place ces cartes constellations. Elles commencent alors à réfléchir ensemble à sa fabrication.

Dans les pratiques de l'atelier, les travailleurs ont souvent recours à des plateaux pour poser leur préparation et les déplacer. Une discussion s'engage entre la chercheure et la monitrice d'atelier présente ce jour-là, sur les quantités d'objets placés sur les plateaux. Il s'agit de définir ensemble les configurations canoniques propres à la restauration. La monitrice signifie qu'on ne peut placer plus de neuf objets sur ces plateaux, que ce soit des petites assiettes pour le fromage ou des soucoupes pour les cafés. La chercheure et la monitrice décident donc de représenter des quantités comprises entre un et neuf. De plus, pour faciliter la perception du lien entre les cartesconstellations et les plateaux sur lesquels seront posés les objets, la forme des cartesconstellations est pensée à partir de la forme des plateaux, soit des rectangles aux angles arrondis. Les plateaux peuvent de cette manière remplir leur rôle de repère spatial pour disposer les objets, en reproduisant les collections témoins organisées des cartes-constellations. Enfin, la chercheure et la monitrice conviennent ensemble de différentes dispositions spatiales selon des quantités croissantes de un à neuf (illustration 1).

Illustration : Les neuf cartes-constellations imaginées.

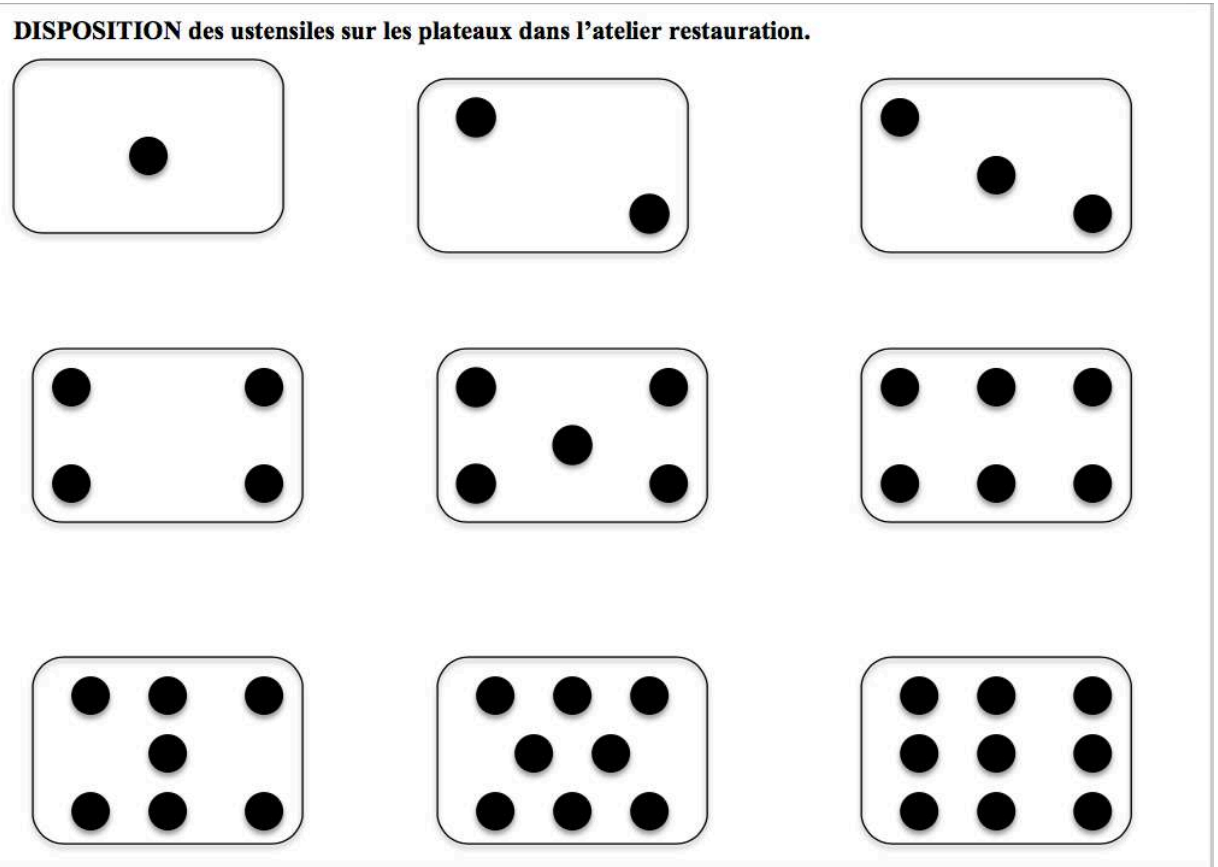

Chaque disposition spatiale s'appuie sur la manière dont peuvent être disposées les petites assiettes à fromage. Chacune des cartes-constellations a la même forme que les plateaux de l'atelier restauration

Reprenons notre situation imaginaire des vingt-cinq petites assiettes à fromage. La monitrice peut tout d'abord donner à Walter trois plateaux et une pile de petites assiettes. Ensuite, elle lui tend la carte-constellation neuf en lui demandant de poser sur le plateau neuf petites assiettes à fromage. Elle réitère l'opération pour le second plateau. Puis, pour le dernier, elle donne cette fois-ci la carte-constellation sept. En 
observant la configuration canonique de la carte-constellation et en se repérant sur l'espace que constitue le plateau, Walter peut ainsi préparer plateau par plateau (d'abord 9 assiettes, puis encore 9 , puis 7) les vingt-cinq petites assiettes à fromage. Par la suite, il a simplement à mettre les fromages, portions de beurre et feuilles de salade dans chacune des assiettes, en utilisant la correspondance terme à terme, qu'il maîtrise. Néanmoins, la chercheure anticipe un obstacle potentiel pour Walter : s'il n'arrive pas à poser directement des objets, par exemple des verres, sur le plateau selon les configurations canoniques des cartes-constellations, quelle solution apporter?

\subsubsection{Les pastilles}

Utiliser les cartes-constellations ne va pas de soi. S'appuyant sur son expérience antérieure, la chercheure imagine des pastilles, c'est-à-dire des ronds noirs de papier plastifiés de la taille d'une soucoupe. Par exemple, Walter a déposé ici (Illustration 2) autant de pastilles que de points figurant sur la carte-constellation, soit neuf: on remarque donc ici qu'il n'a pas besoin de s'appuyer sur l'espace du plateau pour positionner correctement les pastilles sur la table.

Illustration : la carte-constellation neuf est posée sur la table.

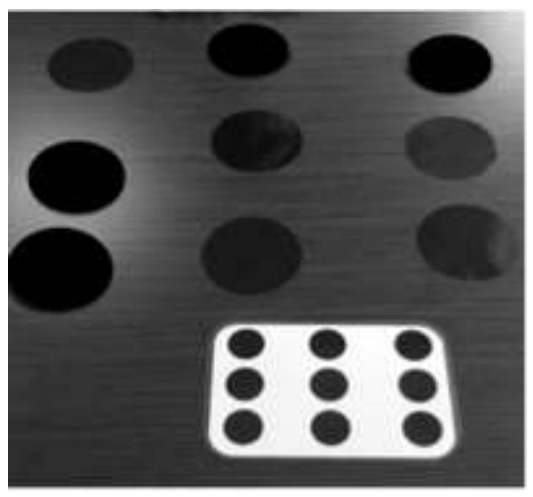

Au-dessus, on aperçoit neuf pastilles disposées, faces noires vers le haut, selon la configuration spatiale de la carte neuf.

Ces pastilles jouent, d'une certaine façon, le rôle de constellation amovible, en étant positionnées avec leur face noire visible selon la configuration canonique d'une des cartes-constellations.

\subsubsection{Des cartes-constellations partagées}

Si les premières interventions de la chercheure, auprès de Walter, consistent à proposer des situations avec des nombres, et d'expérimenter les cartes-constellations et les pastilles, elles permettent aussi aux monitrices de se familiariser avec ces deux outils d'accessibilité en les expérimentant à leur tour dans l'activité de l'atelier. Elles invitent donc le travailleur à les utiliser dès qu'une activité professionnelle nécessite le recours aux nombres. Cette appropriation des monitrices est d'autant plus facile qu'elles y voient rapidement un intérêt. En effet, les cartes-constellations permettent à Walter de travailler seul. Les monitrices peuvent donc accompagner d'autres travailleurs. L'accompagnement de l'atelier est amélioré. La production est facilitée. 
29 À chaque nouvelle rencontre ${ }^{9}$, chercheure et monitrices se nourrissent mutuellement des remarques des unes et des autres. Avant chaque intervention de la chercheure auprès de Walter, les monitrices peuvent ainsi indiquer les réussites et les obstacles rencontrés par ce dernier dans son activité professionnelle. La chercheure peut donc ajuster son intervention. En retour, après l'intervention, la chercheure fait un bilan et indique les stratégies mises en œuvre par le travailleur, lors des situations d'enseignement-apprentissage mettant en jeu des nombres. Les monitrices peuvent, à leur tour, ajuster leur accompagnement dans le contexte professionnel, en prenant en compte les remarques de la chercheure. A chaque fois, les cartes-constellations sont utilisées. Chacun des co-actants agit donc dans son contexte, en ajustant son action à partir des expériences de l'autre. Comme les cartes-constellations assurent une forme de continuité de l'expérience du travailleur, celui-ci utilise une technique utilisant les nombres (par exemple, disposer cinq objets selon la configuration canonique de la carte-constellation cinq), sans être déstabilisé par le changement de milieu.

\subsubsection{Cartes-constellations et évolutions des pratiques}

30 Les semaines passent. Un rythme est trouvé. Monitrices et chercheure se nourrissent réciproquement de leurs actions auprès de Walter. Une ingénierie coopérative émerge, constituée de la chercheure, des monitrices et de Walter. Les monitrices amorcent un questionnement sur l'opportunité d'utiliser les cartes-constellations avec d'autres travailleurs. Très rapidement, au vu des réussites de Walter, les pastilles sont abandonnées. Assurées dans leurs pratiques et entrevoyant les nouvelles possibilités d'actions avec des cartes-constellations, les monitrices s'emparent de ce nouvel outil d'accessibilité.

31 Une des monitrices raconte ainsi, qu'un jour, elle a eu besoin de demander douze compotes à Walter. Les cartes-constellations n'allant que jusqu'à dix, elle a alors spontanément dessiné douze points sur une ardoise. Précisons que c'est en voyant, à plusieurs reprises, la chercheure utiliser ce support, que les monitrices ont décidé d'acheter des ardoises pour l'atelier restauration. Walter n'a eu aucune difficulté pour rapporter les douze compotes en les disposant suivant la configuration canonique dessinée.

Le fait que les cartes-constellations soient entrées pleinement dans les pratiques de la restauration assure donc une dynamique de création collective. Pour l'illustrer, nous relatons dans ce qui suit, comment le travail d'enquête collectif a pu transformer les cartes-constellations en un porte-clés de cartes-constellations.

Ce jour-là, comme à chaque fin de séance, la chercheure commence par faire un bilan de ses observations aux monitrices. Puis, les échanges s'orientent vers un constat collectif : Walter progresse ; les cartes constellations le mettent en situation de réussite.

Le collectif commence alors à réfléchir ensemble à un outil qui faciliterait encore davantage le travail. La chercheure suggère un porte-clés. Dans l'extrait du journal de terrain ${ }^{10}$ ci-dessous, nous prenons connaissance de la réaction des monitrices.

« (...) Les monitrices trouvent l'idée bonne, mais elles me disent alors qu'il n'a pas de ceinture et qu'il y a des règles de sécurité, à savoir, éviter des objets qui pourraient tomber dans la friteuse par exemple, ou attraper une casserole au passage. Puis, une monitrice pense à un collier tels ceux utilisés par certains travailleurs pour garder leurs clés autour du cou. Sa collègue pense à une pince pour l'accrocher dans la poche de la blouse (...)». 
La semaine suivante, la chercheure revient avec le porte-clés des cartes constellations, constitué de petites cartes-constellations, fabriquées sur le même modèle que les grandes (Illustrations 3, 4 et 5). Elle a écrit au dos de chacune, les nombres correspondants. Walter et la monitrice, présente lors de la séance, accueillent ce support très favorablement.

Illustration : Les cartes-constellations et le porte-clés des cartes-constellations.

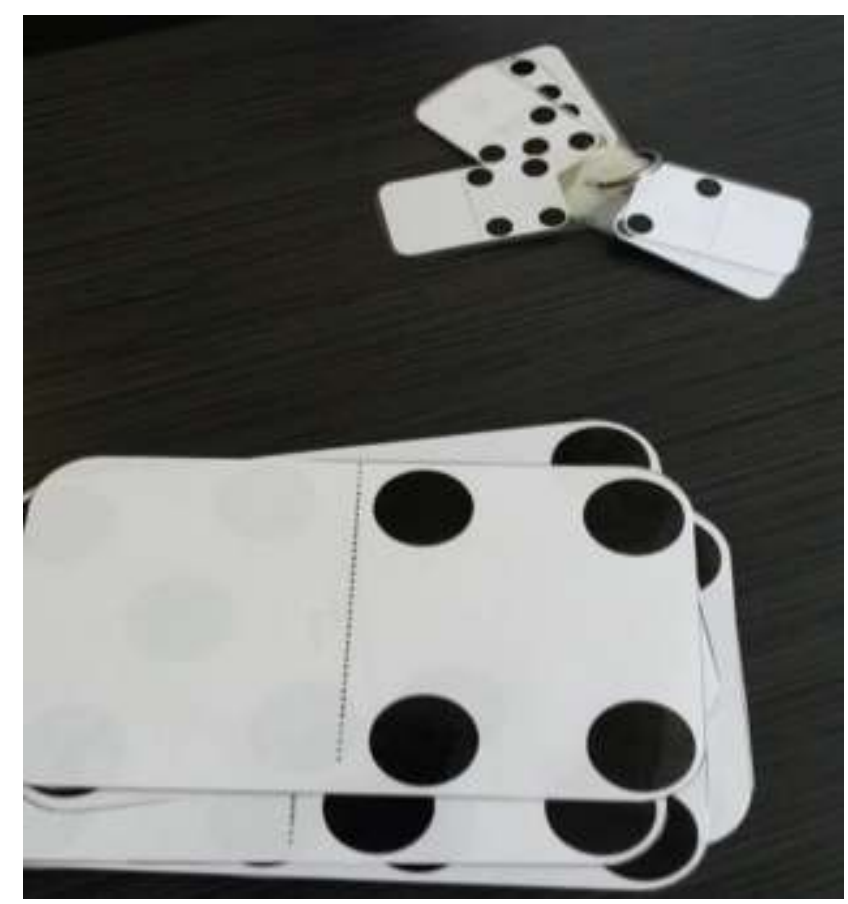

Illustration : Le porte-clés des cartes-constellations, face cartes-constellations.

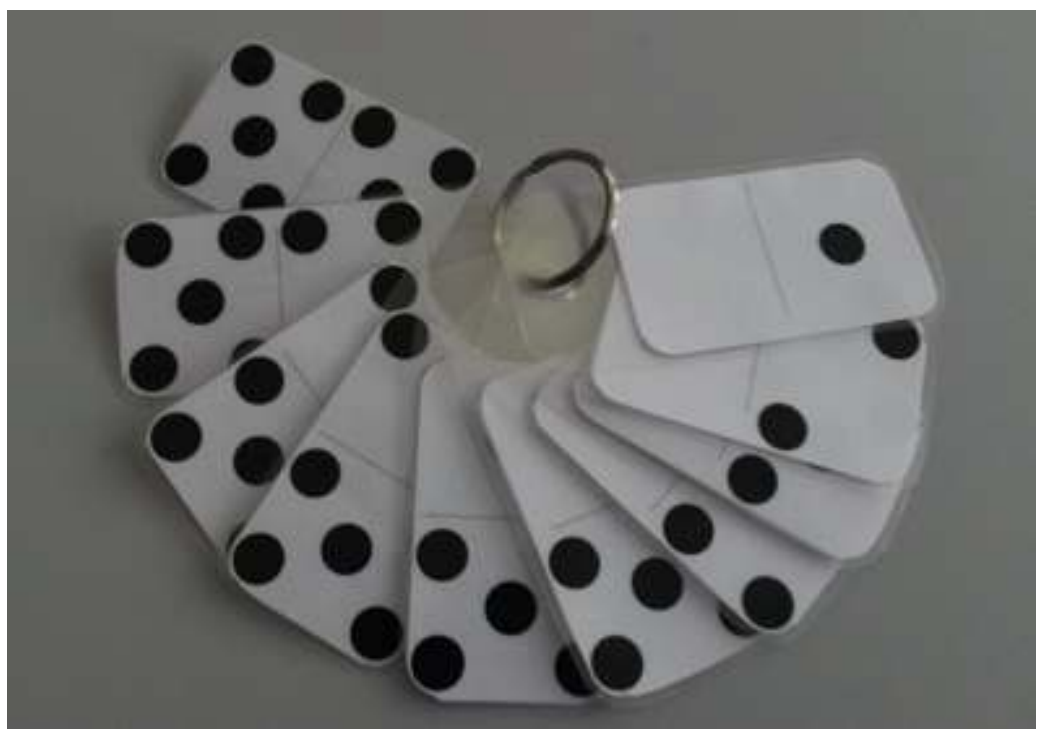

Les configurations canoniques ont évolué pour des configurations du type 5+5. 
Illustration : Le porte-clés des cartes-constellations, face nombres.

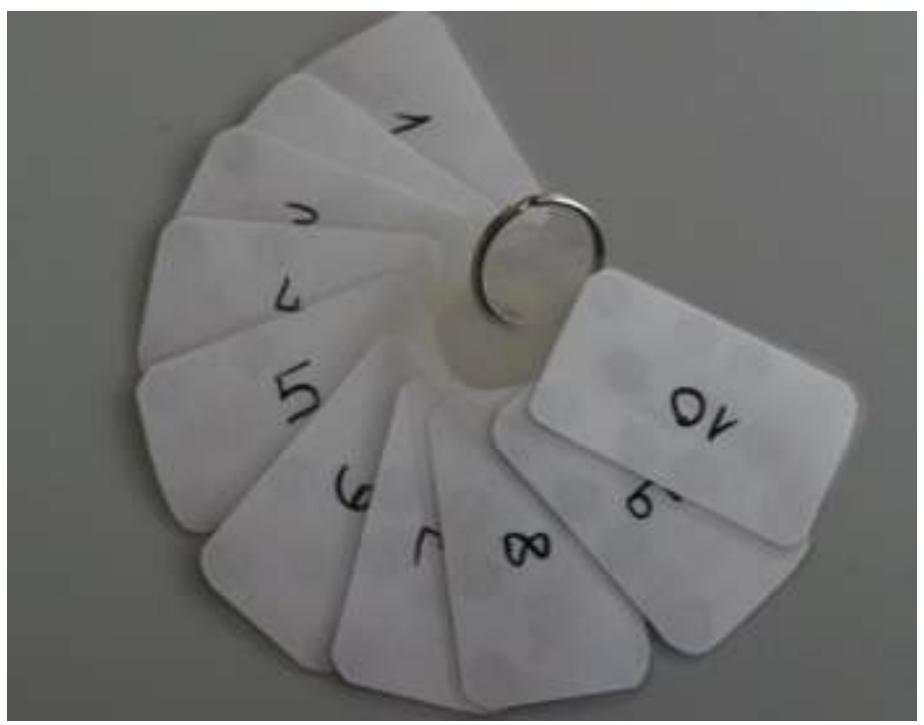

36 L'intervention de la chercheure consiste donc, ce jour-là, à ce que Walter se familiarise avec le porte-clés de cartes-constellations. Ainsi, à l'instar de situations déjà proposées avec les cartes-constellations, la chercheure demande à Walter d'aller chercher sept couteaux (Illustration 6), puis cinq coupes à glace (Illustration 7). À chaque fois, Walter est en réussite.

Illustration : Le porte-clés des cartes constellations et sept couteaux.

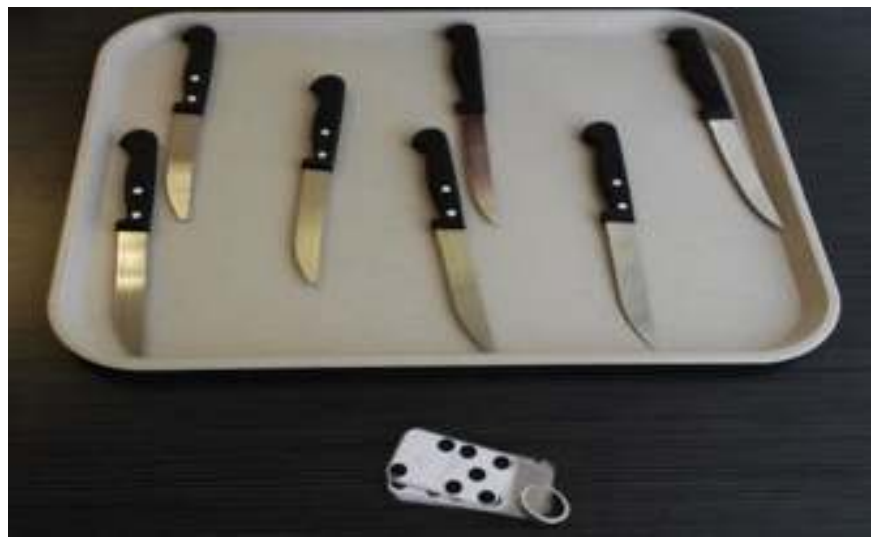


Illustration : Le porte-clés des cartes constellations et cinq coupes à glace.

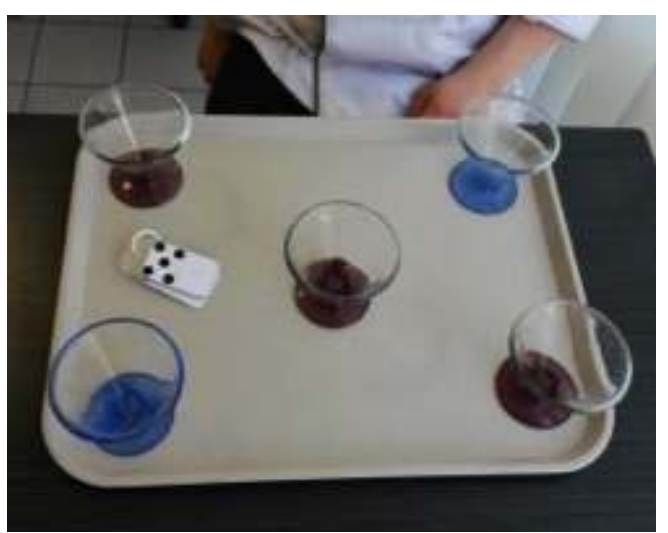

37 À la fin de la séance, lors de l'échange avec la monitrice, cette dernière propose à Walter de garder tout le temps ce porte-clés sur lui, car c'est une aide pour compter. Elle lui suggère même de l'emmener à son domicile. L'implication de la monitrice est complète.

Dès lors, Walter a en permanence ce porte-clés des cartes-constellations autour du cou, sous sa blouse de travail. Il est aujourd'hui capable de dénombrer des collections en utilisant la comptine numérique. Cependant, lorsqu'il se trompe, les monitrices peuvent lui demander de vérifier avec ce porte-clés des cartes-constellations.

Un second porte-clés a été fabriqué pour un autre travailleur. Lors de la troisième réunion de l'ingénierie coopérative, une des monitrices de la restauration a pu dire à propos de ce travailleur : « je pense qu'il a un manque de confiance en lui, du coup, là au moins il est sûr, il va chercher, il y a dix bananes, c'est bon! $»^{11}$ (Tdp.735). En d'autres termes, le porte-clés est entré dans les pratiques de l'atelier. Il permet au travailleur de vérifier seul ses actions, ici aller chercher dix bananes. Convaincues par cet outil d'accessibilité, les monitrices peuvent donc prendre en charge d'autres aspects de la pratique dans leur accompagnement.

\section{Analyse}

Nous allons, dans ce qui suit, produire une redescription en langage théorique de notre exemple emblématique.

41 Pour modéliser les actions conjointes des différents acteurs de cet exemple emblématique, nous utilisons la notion de jeu.

42 Nous modélisons ainsi l'activité précédemment décrite comme deux jeux imbriqués. Le jeu d'ingénierie modélise l'activité des monitrices et de la chercheure, c'est-à-dire le travail d'enquête collectif. Le jeu didactique modélise l'activité du travailleur Walter, des monitrices de la restauration et de la chercheure dans l'atelier. Dans ce jeu d'ingénierie, les monitrices de la restauration et la chercheure construisent ensemble un jeu didactique.

43 Ce préalable effectuée, nous allons maintenant étudier de quelle manière la coopération chercheure/monitrices se construit en nous centrant plus précisément sur le rôle joué par les outils d'accessibilité, notamment les cartes-constellations, dans la mise en place de cette coopération. 


\subsection{Partager des déjà-là pour élaborer des stratégies communes} projet. Avec la chercheure, elles partagent donc, d'emblée, quelques premiers éléments d'un arrière-plan commun: 1/ la chercheure va intervenir auprès de Walter, en lui proposant des situations d'enseignement-apprentissage organiquement reliées à l'activité professionnelle; 2 / les monitrices et la chercheure vont devoir travailler ensemble pour co-élaborer ces situations, chacune avec ses connaissances propres ; 3/ elles vont aussi travailler ensemble pour assurer une continuité des situations d'enseignement-apprentissage avec celles de la pratique. C'est le contrat didactique initial partagé du jeu d'ingénierie. Nous l'appelons, dans la suite de notre propos, contrat de coopération. pratique du travailleur («permettre à Walter de constituer seul des collections d'objets »), dans un même temps, un jeu d'ingénierie est inauguré avec un autre problème commun à résoudre : « travailler ensemble, sachant que la chercheure et les monitrices de la restauration n'ont jamais coopéré ». Ce problème se caractérise par une structure particulière, qu'on peut considérer comme le milieu de l'action, c'est-àdire «ce avec quoi il y a à faire pour avancer dans la résolution d'un problème. Le milieu désigne ainsi la structure symbolique du problème que l'élève doit travailler. » (CDpE, 2019, p. 594). Nous pouvons voir ce milieu comme ce qui est à-connaître.

Au départ, pour travailler à ce problème, chacun des acteurs dispose d'un système de connaissances déjà-là qui lui est propre. Chacun est connaisseur pratique d'une certaine pratique. De tels systèmes pourraient être formulés génériquement ainsi : je suis connaisseur d'une pratique; j'ai des habitudes dans ma pratique; j'ai des conceptions sur ma pratique; je suis dans une institution, c'est-à-dire qu'il existe des habitudes institutionnelles qui me font agir. Dans la mesure où les acteurs vont être amenés à coopérer, nous appréhendons mieux en quoi la visite des ateliers par la chercheure est l'occasion d'enrichir l'arrière-plan commun en genèse. La chercheure, non connaisseuse pratique de la restauration, prend ainsi connaissance de l'activité réelle de l'atelier, et notamment de l'incidence que peut avoir la production de l'atelier sur l'accompagnement des travailleurs. Elle appréhende donc quelques aspects du système de connaissances déjà-là des monitrices de l'atelier, connaisseuses pratiques de l'atelier restauration (par exemple, comme tout service de restauration, il faut veiller à respecter les normes sanitaires. Ces normes agissent sur l'activité professionnelle des moniteurs et des travailleurs).

47 La chercheure cherche donc du connu, qui lui permette d'exercer ses capacités, dans un environnement inconnu (à-connaître). Pour ce faire, la chercheure tente de repérer des points d'appui, du connu, dans le milieu de l'atelier restauration. Ainsi, en observant la pratique professionnelle des travailleurs, la chercheure, connaisseuse pratique du métier d'enseignante spécialisée, repère des situations dans lesquelles les travailleurs doivent recourir aux nombres : comme on l'a vu, Walter a dû dénombrer vingt-sept prunes. Son collègue a préparé vingt-cinq assiettes à fromage.

48 Cette recherche est donc orientée, pour la chercheure, de deux façons ;

- d'une part, par un système de connaissances relevant du déjà-là : chercher des situations « raisonnablement analogues » à sa pratique antérieure d'enseignante spécialisée ;

Questions Vives, $N^{\circ} 31$ | 2019 
- d'autre part, par le contrat de coopération monitrices/chercheure défini plus haut. des moniteurs et des travailleurs de l'atelier restauration, tout en se centrant plus particulièrement sur l'activité de Walter, le travailleur auprès duquel elle va agir par la suite. Cette prospection porte ses fruits, puisqu'elle entrevoit un problème possible: « permettre à Walter de constituer seul des collections d'objets». Les savoirs en tant que puissance d'agir sont des solutions à ce problème. Aussi, chercher à résoudre ce problème c'est chercher à envisager des situations d'enseignement-apprentissage dans lesquelles la chercheure et les monitrices vont faire travailler les nombres à Walter. Il s'agit donc d'un jeu didactique.

\subsection{Des actions conjointes différées}

La solution trouvée au problème « permettre à Walter de constituer seul des collections d'objets » est matérialisée par deux outils d'accessibilité : les cartes-constellations et les pastilles. On l'a vu, la stratégie envisagée collectivement est de proposer à Walter de s'appuyer sur les configurations canoniques des cartes constellations pour constituer des collections d'objets.

51 Par la suite, le collectif poursuit ce travail d'enquête collectif en affinant sa stratégie commune. Au départ, c'est la chercheure qui tente d'élucider des aspects du problème auquel elle n'a pas accès d'emblée. En appui sur ses connaissances pratiques d'enseignante spécialisée, elle demande aux monitrices de réfléchir, par exemple, à des situations dans lesquelles les outils d'accessibilité pourraient être utilisés par Walter, sans entraver l'activité de l'atelier. De cette façon, la chercheure invite les monitrices à partager leurs déjà-là, au nom du principe de symétrie propre à celui ou celle qui veut mener un travail d'enquête collectif prenant la forme d'une ingénierie coopérative. Ce faisant, les monitrices proposent des modifications de leur travail commun, liées à leur propre expérience. Ainsi, elles prennent graduellement une responsabilité de plus en plus grande dans leur travail commun Peu à peu, leur assomption des problèmes travaillés ensemble s'accroît. En faisant part de leurs pratiques, les monitrices continuent de contribuer à l'élaboration d'une stratégie commune, qui résultera de la prise en compte effective et collective du milieu, de la structure particulière du problème. coopérative, s'entendent donc pour ne pas agir en même temps auprès de Walter. La chercheure le fera jouer en dehors du "rush" de l'activité, sur un temps déterminé, à raison d'une fois par semaine, c'est-à-dire que lors de ses interventions, elle proposera des situations d'enseignement-apprentissage dans lesquelles Walter utilisera les cartesconstellations. Les monitrices de la restauration feront jouer ce même jeu didactique au cours de l'activité professionnelle de Walter, c'est-à-dire qu'elles l'inciteront à utiliser les cartes constellations, dès que la situation le nécessitera.

Ainsi, dans le travail d'un problème spécifique (construire le jeu didactique « permettre à Walter de constituer seul des collections d'objets »), mené ensemble par le collectif, les monitrices et la chercheure définissent une forme didactique générique de leurs actions à venir auprès de Walter. Cette forme didactique crée une sorte d'alternance entre un entraînement protégé (en dehors de la contrainte de la production) dans lequel Walter s'approprie certaines stratégies, et une mise en œuvre effective 
accompagnée, au travail, de ces stratégies. Une partie importante des situations proposées dans le cadre de l'entraînement protégé pourra se faire en s'appuyant sur les obstacles rencontrés par Walter lors de l'activité professionnelle. Le collectif pourra réfléchir ensemble à des solutions. La chercheure pourra ensuite les proposer dans un cadre non soumis à la production et recueillir ainsi un certain nombre d'informations utiles quant au travail d'enquête collectif, la visée commune étant d'amener Walter à dépasser l'obstacle. La continuité de l'expérience sera ainsi mieux assurée, non seulement pour Walter, mais encore pour les monitrices et la chercheure.

Examinons, maintenant, plus particulièrement la fonction des outils d'accessibilité dans le jeu didactique joué dans l'atelier.

\subsection{Le rôle des outils d'accessibilité}

\subsubsection{Une hybridation matérielle de déjà-là différents}

Considérant les cartes-constellations et des pastilles, à bien y regarder, ces outils d'accessibilité peuvent être vus comme la résultante matérielle de la rencontre de systèmes de connaissances initiaux (de déjà-là) différents. D'une certaine façon, ils peuvent être vus comme une hybridation matérielle des déjà-là des monitrices et de la chercheure. En effet, utiliser les configurations canoniques pour constituer des collections d'objets ou utiliser des pastilles pour s'appuyer sur l'équipotence de collections provient du connu de la chercheure.

La taille des outils, leur facilité de manipulation et le fait que ces outils soient plastifiés, résultent du connu des monitrices de la restauration. Enfin, la forme des cartes identiques à la forme des plateaux, la taille des pastilles, et les dispositions spatiales possibles des objets sur un plateau, définies ensemble, résultent du travail d'enquête collectif et donc de la transformation de l'arrière-plan commun dans la problématisation et la résolution du problème

Nous résumons l'hybridation dans « l'équation » suivante : « déjà-là chercheure + déjàlà des monitrices + construction commune du problème à résoudre $=$ quelque chose de nouveau». Quelque chose de nouveau, parce qu'il ne s'agit pas de la simple juxtaposition de deux déjà-là. Ceux-ci doivent en effet s'accommoder ensemble au problème qu'il faut d'abord construire, puis résoudre. Les deux outils d'accessibilité (les cartes constellations et les pastilles) peuvent d'une certaine façon être vus comme la résultante tangible des premiers pas de la coopération.

\subsubsection{Des liens}

Le jeu didactique "permettre à Walter de constituer seul une collection d'objets " consiste à ce que le travailleur utilise de manière adéquate les outils d'accessibilité que sont les cartes-constellations et les pastilles. Walter doit ainsi constituer une collection d'objets en s'aidant de ces outils d'accessibilité, quand il rencontre une situation nécessitant le recours au dénombrement. En d'autres termes, Walter peut utiliser ces outils d'accessibilité, comme stratégies, pour aborder un problème rencontré, dans toute situation de dénombrement.

Dans l'idée d'une forme didactique "en alternance ", comme nous avons pu le dire plus haut, un processus dynamique se dessine, dans lequel un arrière-plan commun et un système stratégique commun s'élaborent. Aussi, même si les monitrices et la 
chercheure n'agissent pas en même temps, ce partage de connus permet au fur et à mesure de proposer des stratégies de plus en plus efficaces et donc de faire jouer des jeux didactiques de plus en plus adéquats aux situations, dans une sorte de processus continu.

Ici, l'outil d'accessibilité, les cartes-constellations, constitue un invariant de ce processus. Plus précisément, ce sont les formes symboliques et sémiotiques qui sont invariantes, puisque nous avons vu dans un paragraphe précédent que les cartes constellations en tant que telles vont se transformer en un porte-clés de cartesconstellations. Cet outil d'accessibilité constitue la forme matérielle de l'arrière-plan commun aux trois instances monitrices/chercheure/Walter.

61 À chaque séance de l'activité modélisée comme jeu d'ingénierie, les acteurs font part de la manière dont ces outils d'accessibilité ont permis à Walter d'agir adéquatement ou non. Les cartes-constellations permettent donc aux acteurs de partager des stratégies gagnantes ou non, mises en œuvre par Walter dans leur contexte. Les cartesconstellations revêtent la forme d'un milieu matériel sur lequel le travail d'enquête collectif se cristallise.

Dans le jeu didactique, les cartes-constellations matérialisent le déjà-là de Walter construit dans l'action conjointe didactique antérieure, qu'il s'agisse de l'action conjointe Walter/monitrices ou de l'action conjointe Walter/chercheure. Elles permettent à Walter d'initier des stratégies lui permettant de gagner au jeu didactique. $\mathrm{Au}$ fur et à mesure, Walter sait que l'utilisation de ces cartes constellations peut l'aider à résoudre un problème auquel il est confronté.

\section{Synthèse}

63 Nous avons produit un schéma (schéma 1) embrassant dans un processus dynamique, la construction d'un arrière-plan commun et d'un système stratégique commun, à partir de l'outil d'accessibilité, les cartes-constellations. 
Schéma : Processus dynamique de construction d'un arrière-plan commun et d'un système stratégique commun

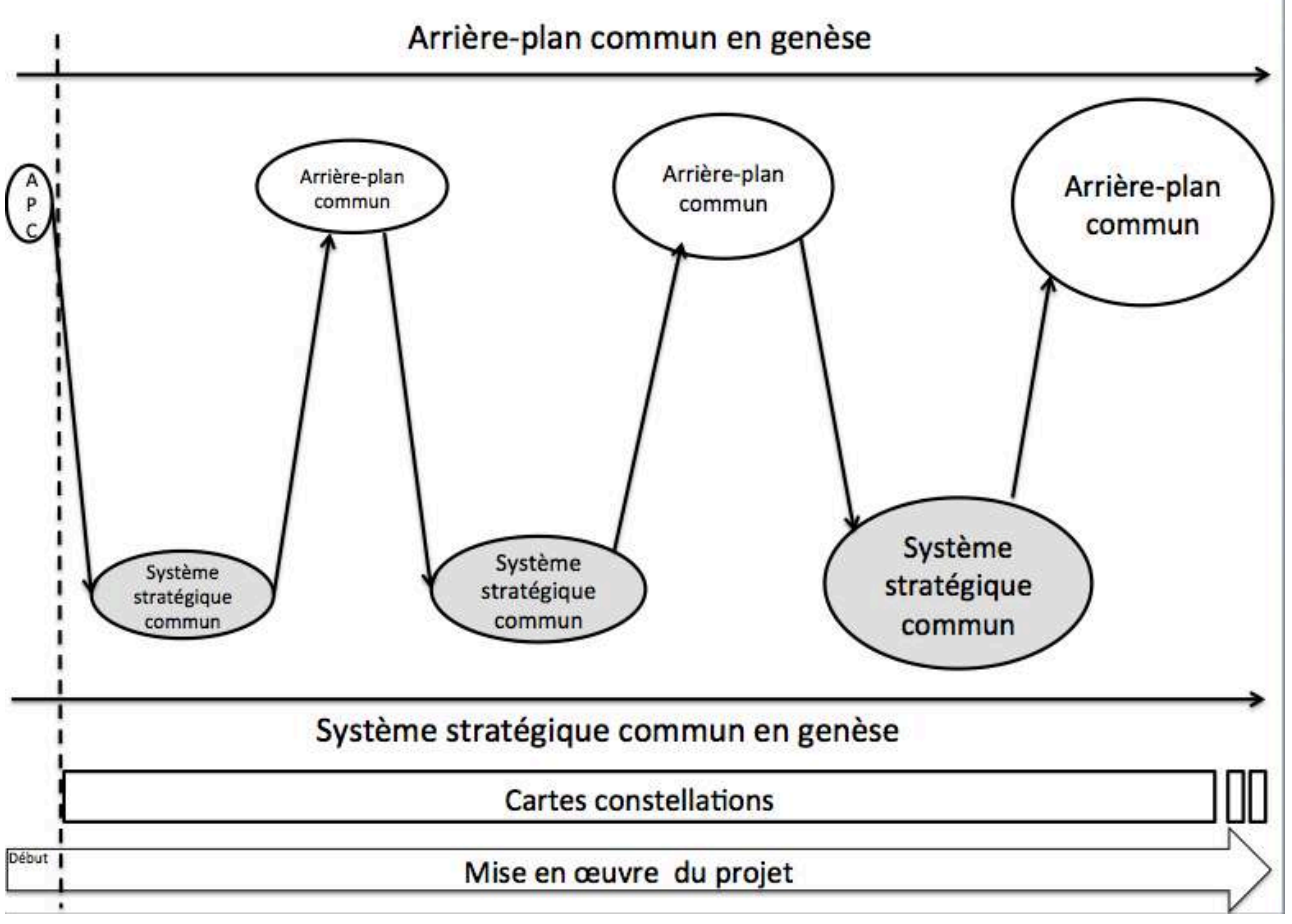

Dans le travail d'enquête collectif, la chercheure et les monitrices ont échangé pour alimenter l'arrière-plan commun en genèse. C'est pour cette raison qu'est figurée sur ce schéma (schéma 1) une succession d'arrière-plans communs d'aires de plus en plus importantes, au fur et à mesure de l'avancée du projet. Au début, il existe un faible arrière-plan commun (APC sur le schéma ${ }^{12}$ ) : travailler ensemble auprès de Walter dans son atelier.

Dans leurs échanges, les monitrices d'atelier et la chercheure définissent ensemble un système stratégique visant à faire jouer le jeu didactique. Ce système stratégique en genèse s'appuie sur l'arrière-plan commun également en genèse. En retour, il est éprouvé dans le jeu didactique in situ. Il nourrit alors l'arrière-plan commun. Aussi, il apparaît sous la succession des arrière-plans communs, une succession de systèmes stratégiques communs (en gris), également en croissance.

Enfin, sur ce schéma, l'encadré intitulé « cartes-constellations » signifie que les formes symboliques des cartes-constellations constituent un invariant du processus. A chaque étape du processus, le nourrissage de l'arrière-plan commun offre donc des nouveaux possibles à chacun, en permettant d'aborder de nouveaux problèmes. Le moteur de ce processus dynamique tient aux réussites successives que nous formulons comme suit: " on garde ce qui marche parce qu'on croit en ce qui marche». La réussite assure l'engagement qui assure la réussite, etc.

Un exemple concret de cette forme de dynamique est l'évolution des cartesconstellations. La réussite de la coopération, telle qu'elle s'exprime dans les cartesconstellations, va engendrer un nouveau travail d'enquête collectif qui va conduire à l'invention d'un outil d'accessibilité matériellement plus adéquat encore à l'atelier de la restauration, le porte-clés de cartes-constellation. 


\section{Discussion}

\subsection{Un espace pour voir comme autrui, base de toute coopération}

68 Dans la mise en place de l'ingénierie coopérative, l'enjeu premier était que les acteurs apprennent à travailler ensemble, à agir conjointement. Il s'agissait, en d'autres termes, de construire un collectif de pensée (Fleck, 2005), c'est-à-dire un style de pensée qui amène les membres de l'ingénierie à une vision et une perception concrète communes des problèmes qu'ils avaient à construire et résoudre (Sensevy, 2011, 2016). Or, au départ du projet, monitrices et chercheure sont dans des collectifs de pensée distinct. Chaque collectif a un style de pensée, et donc un arrière-plan, un voir-comme (Wittgenstein, 2004) qui lui est propre. Dès lors, dans les premiers pas de la coopération, il était essentiel que monitrices et chercheure travaillent à partager leurs arrière-plans respectifs.

69 La chercheure, garante du projet, est arrivée dans l'atelier de la restauration avec un style de pensée qui lui était propre. Aussi, en observant Walter en situation professionnelle, elle a vu l'activité du travailleur comme une activité de comptage. Dans ses observations, elle a donc saisi une autre grammaire de la pratique de Walter à laquelle les monitrices ne pouvaient accéder, ces dernières ne disposant pas du même arrière-plan. Le premier effet d'une coopération entre des acteurs disposant de styles de pensée différents est donc d'appréhender différemment une même situation professionnelle : ici, Walter préparant des desserts. Le partage d'arrière-plan a pour conséquence que monitrices et chercheur voient désormais cette même situation comme une activité professionnelle en restauration et comme une activité de comptage. Monitrices et chercheure construisent conjointement une nouvelle grammaire, une nouvelle logique de la pratique de Walter. Ce faisant, elles peuvent envisager d'agir autrement parce que conjointement, en réfléchissant à des stratégies communes.

70 Cet assertion va dans le sens de Fleck, qui considère qu' « un collectif de pensée est toujours en germe dès que deux ou plusieurs personnes échangent des idées » (Fleck, 2005, p. 81). Plus généralement, une même situation est toujours appréhendée différemment selon un style de pensée donnée. Mais dès lors qu'il existe un espace pour échanger sur ces différences de perceptions, pour voir comme autrui, telle l'ingénierie que nous venons de décrire, des possibilités de coopération peuvent toujours émerger, considérant qu'aussi éloignés soit nos perceptions, nos conceptions et nos affects, fondamentalement, en tant qu'humain nous avons quelque chose à partager (Bazin, 2008).

\subsection{Les cartes-constellations comme œuvre commune}

71 Le collectif de pensée en genèse (monitrices et chercheure) partage un but commun : permettre à Walter d'agir plus adéquatement dans l'atelier restauration. Dans notre exemple, nous avons vu que pour y parvenir, le collectif de pensée se centre sur une action a priori spécifique au style de pensée de la chercheure (le comptage avec des cartes-constellations). Néanmoins, ce n'est pas pour autant que cette action dénature la logique première de la pratique de la restauration, puisque les monitrices assument complétement le travail d'enquête. Cette assertion est d'autant plus vraie, qu'un 
nouveau style de pensée a émergé - comme nous l'avons décrit ci-dessus - et qu'il invite à voir dorénavant, par exemple, les activités de Walter comme une activité de restauration et de comptage. La coopération, cette idée de faire œuvre commune, est donc facilitée, car il s'agit d'un échange "gagnant-gagnant " (Sennett, 2014, p. 334), c'est-à-dire un échange dans lequel au travers de négociations, un équilibre peut se construire petit à petit, chacun (monitrices, chercheure, et même Walter) trouvant un intérêt dans la coopération. Mais quelle est exactement cette œuvre commune ? Quelle forme revêt-elle?

Il se trouve que, dans notre cas, cette œuvre (commune) est matérialisée par un objetsolution: les cartes-constellations. Ces cartes-constellations en tant que signes (ici écrits) véhiculent elles aussi un style de pensée, ou dans une perspective wittgensteinienne, un jeu de langage/forme de vie ${ }^{13}$ (Wittgenstein, 2004). Sensevy écrit à propos de ces jeux de langage/forme de vie que c'est « une manière de produire un certain système de signes dont la reconnaissance permet de jouer le jeu » (Sensevy, 2011, p. 57). Dans notre cas, ce système de signes vise à permettre à Walter d'agir dans l'atelier restauration. Produire un certain système de signes pour des acteurs, qui au départ ne partagent pas le même style de pensée, devient alors le nouvel enjeu de l'ingénierie coopérative. Cependant, nous pouvons aisément concevoir que les cartesconstellations entretiennent une familiarité plus proche avec le style de pensée, initial de la chercheure qu'avec celui initial des monitrices. Mais l'essentiel ici, selon nous, tient au fait que pour l'ensemble des membres de l'ingénierie, une prise de conscience s'est faite (pour la chercheure autant que pour les monitrices) : la production commune de systèmes symboliques a constitué à la fois une manière de travailler le problème fondamental rencontré (permettre à Walter d'agir) et une manière de faire cuvre commune.

La place de l'écrit, et des formes symboliques qu'il permet de produire, va donc être questionnée par l'ensemble des acteurs de l'ingénierie coopérative et ce quel que soit le rapport effectif à l'écrit des travailleurs (lecteurs ou non lecteurs).

$\mathrm{Du}$ point de vue des travailleurs, et notamment celui de Walter, les cartesconstellations lui permettent de construire un nouveau rapport au nombre. En effet, elles émettent un certain nombre de signes, dans un certain style de pensée (celui des monitrices et de la chercheure), qui permet à Walter d'agir avec une forme de certitude dans l'atelier. Cette action de Walter est possible parce qu'il a appris à décrypter et reconnaître adéquatement les signes produits via les cartes constellations par les monitrices et la chercheure. Walter est donc entré lui aussi dans le style de pensée de l'ingénierie, ses actions contribuant directement au travail d'enquête collectif. Pour abonder dans ce sens, les cartes-constellations ont été dupliquées par la suite pour servir à un travailleur collègue de Walter. De cette façon, le collectif comprend mieux les liens qu'entretiennent les personnes en situations de handicap intellectuel et l'écrit, et surtout les possibilités que l'écrit offre, via la mise en place d'outil d'accessibilité, résultante d'une hybridation de style de pensée, telles que les cartes constellations. En cela, les outils d'accessibilité, d'une manière générale, sont l'inscription matérielle de l'œuvre commune de l'ingénierie coopérative de l'ESAT. 


\section{Conclusion}

Dans cet article, nous nous sommes attachés à montrer $1 /$ ce qu'il se passait lorsque des acteurs de cultures différentes apprenaient à travailler ensemble et 2/ ce que cette coopération inédite transformait. Les monitrices et la chercheure ont mené un travail d'enquête anthropologique (Sensevy, 2015, 2016). Elles ont essayé de comprendre ce qui pouvait entraver l'action de Walter. Elles ont essayé de comprendre réciproquement leur grammaire de la pratique. Ce faisant, en parallèle, elles ont mené un travail d'enquête ingénierique en transformant l'environnement de Walter par la mise en place des cartes constellations. Mais, elles ont aussi peu à peu mieux compris leurs pratiques qu'elles ont transformées petit à petit, jusqu'à incorporer (Billeter, 2014) un nouveau style de pensée. En témoignent notamment les deux faits suivants: la chercheure découvre et prend en compte les contraintes de production d'un atelier; la monitrice dessine douze points sur une ardoise pour demander à Walter d'apporter des compotes.

76 Au-delà de ces constats locaux, la transformation semble, à ce jour, beaucoup plus profonde. Nous avons vu que l'œuvre commune de l'ingénierie coopérative était de réfléchir à la place de l'écrit et des formes symboliques dans les activités professionnelles des travailleurs d'ESAT. De cette façon, en reconsidérant l'écrit dans ses pratiques, le collectif a été amené à reconsidérer le handicap. Face à une situation rendue complexe par le fait que nous ne pouvons pas interpréter les signes adéquatement ou accéder à la logique de la pratique à laquelle nous sommes confrontés, nous exprimons tous les mêmes difficultés. Nous sommes alors, d'une certaine manière, en situation de handicap. Plus spécifiquement, examinons ce qu'est l'écrit, en reprenant les propos de Goody (2012) décrivant ainsi la découverte de l'écriture dans l'histoire de l'humanité :

Bien sûr, il existait d'autres inventions du point de vue des modes de communication, avant l'invention de l'écriture. Il y eut, par exemple, toute la production de l'art rupestre, surtout en France. Mais, il faut toujours rappeler l'importance de l'écriture, qui est une invention humaine incomparable. C'est au fondement de ce que nous sommes tous, et ici aujourd'hui à l'université... c'est vrai qu'on donne des cours à l'oral, mais tout ce que nous faisons est en fait fondé sur l'écrit! Vous avez tous un bout de papier en main; nous sommes comme « emboîtés » dans les sociétés de l'écrit. Mais, il faut rappeler d'abord que si c'est le langage qui nous a fait « humains », c'est l'écriture qui nous a fait, pourrait-on dire en français, « civilisés ». ${ }^{14}$

77 Certaines difficultés des personnes en situation de handicap ne pourraient-elles pas provenir du fait que nous sommes des humains, « emboités » dans une société où l'écrit est omniprésent? Mobiliser l'écrit dans les pratiques, quelles qu'elles soient, s'inscrire dans les formes symboliques qu'il fait vivre, ne serait-ce pas permettre, à tout un chacun, d'entrer dans la culture humaine? Quoi qu'il en soit, en réfléchissant aux symboles, à l'écrit, l'ingénierie coopérative contribue au développement des puissances d'agir en situation de tous et de chacun. C'est un dispositif émancipateur. Aussi, initier d'autres ingénieries coopératives travaillant à des problèmes attachés à des pratiques nous semblerait fructueux pour qui veut travailler à ce que notre société devienne concrètement une société pour tous et pour toutes, une société inclusive. 


\section{BIBLIOGRAPHIE}

Baxandall, M. (1991). Les Formes de l'intention. Nîmes : Jacqueline Chambon.

Bazin, J. (2008). Des clous dans la Joconde. L'anthropologie autrement. Toulouse : Anacharsis.

Billeter, J. F. (2014). Un paradigme. Paris : Allia.

Brissiaud, R. (2014). Pourquoi l'école a-t-elle enseigné le comptage-numérotage pendant près de 30 années? Une ressource à restaurer : un usage commun des mots grandeur, quantité, nombre, numéro, cardinal, ordinal, etc., Repéré à http://www.cfem.asso.fr/debats/premiers-apprentissagesnumeriques

Collectif Didactique pour Enseigner (CDpE). (2019). Didactique pour Enseigner. Rennes : Presse Universitaire de Rennes.

Daguzon, M., Marlot, C., Toullec-Théry, M. (2017). Processus de co-construction et rôle de l'objet biface en recherche collaborative. Phronesis, 6, (1-2), 21-34.

Davydov, V. V. (1990). Types of generalisation in instruction: Logical and psychological problems in the structuring of scholl curricula (Soviet studies in mathematics education, vol. 2; J.

Kilpatrick, Ed., J. Teller, Trans.). Reston, VA: National Council of Teachers of Mathematics [Original work published 1972].

Desgagné, S. et al. (2001). L'approche collaborative de recherche en éducation : un rapport nouveau à établir entre recherche et formation. Revue des sciences de l'éducation, 27(1), 33-64.

Dewey, J. (2014). Reconstruction en philosophie. Malesherbes : Gallimard.

Engeström, Y., (2011). Théorie de l'activité et management. Management\&Avenir 2 (42), p. 170-182. doi $10.3917 / \mathrm{mav} .042 .0170$

Fleck, L. (2005). Genèse et développement d'un fait scientifique. Paris: Les Belles lettres. Gelman, R., Gallistel, C. R. (1978). The child's understanding of number. Cambridge (MA), Harward University Press.

Goody, J. (2012, septembre). Jack Goody et l'empire de la littératie. Repéré à https://www.canal-u.tv/ video/canal_socio/jack_goody_et_l_empire_de_la_litteratie_version_integrale.11211

Goody, J. (2017). La raison graphique. La domestication de la pensée sauvage. Paris: Les éditions de Minuit

Ilyenkov, E. (1982). The Dialectics of the Abstract and the Concrete in Marx's Capital. Moscow : Progress Publishers Kosík, K. (1988). La Dialectique du concret. Paris : Editions de la Passion.

Joffredo-Le Brun, S. (2016). Continuité de l'expérience des élèves et systèmes de représentation en mathématiques au cours préparatoire : une étude de cas au sein d'une ingénierie coopérative. (Thèse de doctorat, Université de Bretagne occidentale, Brest). Repéré à https://tel.archives-ouvertes.fr/ tel-01495980/document.

Leutenegger, F. (2000). Construction d'une Clinique pour le didactique. Une étude des phénomènes temporels de l'enseignement. Recherches en didactique des mathématiques, 20, (2), 209-250.

Malinowski B. (1963). Les Argonautes du Pacifique occidental. Paris : Gallimard. 
Morellato, M. (2017). Travail coopératif entre professeurs et chercheurs dans le cadre d'une ingénierie didactique sur la construction des nombres : conditions de la constitution de l'expérience collective. (Thèse de doctorat, Université de Bretagne occidentale, Brest). Repéré à https://tel.archivesouvertes.fr/tel-01591957/document.

Perraud, C. (2018a). Une ingénierie coopérative dans un Établissement et service d'aide par le travail (ESAT) : un collectif pour penser l'accompagnement. Dans Guirimand, N., Mazereau, P. et Leplège, A. (dir.) Les nouveaux enjeux du secteur médico-social. Décloisonner \& Coordonner les parcours de vie et de soin. (p.201-216.). Nîmes : Champs Social Édition.

Perraud, C. (2018b). Une ingénierie coopérative : des travailleurs, des professionnels et un chercheur dans le secteur du travail protégé (ESAT). Une enquête collective pour une amélioration des pratiques. Thèse de doctorat. Université de Bretagne occidentale, Brest, France.

Perraud, C. (2019). Une ingénierie coopérative en ESAT, quand travailler ensemble rend capable de créativité. L'exemple emblématique « une idée farfelue ». La nouvelle revue - Éducation et société inclusives, 85, 277-297.

Sennett, R. (2014). Ensemble, Pour une éthique de la coopération. Paris : Albin Michel.

Sensevy, G. (1999). Éléments pour une anthropologie didactique. Note pour l'habilitation à diriger des recherches. Marseille : Université de Provence. Repéré à http://python.espe-bretagne.fr/ sensevy/Sensevy\%20(1999)\%20Elements_anthropologie_action_didactique.pdf

Sensevy, G. (2007). Des catégories pour décrire et comprendre l'action didactique. Dans G. Sensevy, \& A. Mercier (Ed.), Agir Ensemble. L'action didactique conjointe du professeur et des élèves dans la classe (p. 13-49). Rennes : PUR.

Sensevy, G. (2011). Le sens du savoir. Eléments pour une théorie de l'action conjointe en didactique. Bruxelles : De Boeck.

Sensevy, G. (2013). Pourquoi Dewey. Quelques remarques introductives. Dans H.-L. Go (dir.), Dewey penseur de l'éducation (p. 9-30). Nancy : Presse universitaires de Lorraine.

Sensevy, G., Forest, D., Quilio, S., \& Morales G. (2013). Cooperative engineering as a specific design-based research. ZDM The International journal on Mathematics education. 45(7), 1031-1043. Repéré à: http://python.espe-bretagne.fr/sensevy/ ZDM\%20Sensevy\%20et\%20al\%202013\%20Cooperative\%20Engineering.pdf

Sensevy, G., Gruson, B. Forest, D. (2015). On the nature of the semiotic structure of the Didactic Action: the Joint Action Theory in Didactics Within a Comparative Approach. Interchange, 46 (4), 387-412. doi 10.1007/s10780-015-9266-2

Sensevy, G. (2016). Apprendre : faire apprendre. Revue française de pédagogie, 192, 109-120. Repéré à http://python.espe-bretagne.fr/sensevy/Sensevy(2015)ApprendreFaire\%20apprendre.pdf

Sensevy, G. (2017). Séminaire « Action ». Recueil inédit, Université de Bretagne Occidentale.

Wittgenstein, L. (2004). Recherches philosophiques. Paris : Gallimard.

Wittgenstein, L. (2006). De la certitude. Paris : Gallimard.

\section{NOTES}

1. L'Apei Ouest 44 est une association gestionnaire du secteur médico-social de parents de personnes en situation de handicap intellectuel. 
2. Nous entendons par problème «toute situation qui confronte une personne à une difficulté d'action » (Sensevy, 2017).

3. Dans notre étude nous avons défini comme "outil d'accessibilité », un support écrit ayant vocation à rendre accessible à tous (lecteurs ou non lecteurs) une situation ou une pratique. Les cartes-constellations rendent accessible la pratique du recours au comptage.

4. Un exemple emblématique a vocation de devenir à terme un exemple exemplaire " exemplar» (Kuhn, 1990), c'est-à-dire « des solutions de problèmes concrets, acceptées par le groupe comme paradigmatiques, au sens tout à fait usuel du terme "Sensevy, 2011, p.207). Outre sa fonction didactique (transmettre une pratique), l'exemple exemplaire a une fonction épistémologique: il permet de caractériser une pratique et de la comprendre.

5. Nous considérons comme connaisseur pratique « celui qui a longuement travaillé une pratique de la culture, et qui est donc capable de l'accomplir avec art, qu'il soit jardinier, musicien, mathématicien, ébéniste, danseur, plombier, etc. Le connaisseur pratique se rend ainsi de plus en plus savant de cette pratique » (CDpE, 2019).

6. «Pour ne prendre qu'un exemple trivial, quelqu'un qui sait où est la boulangerie ira plus vite acheter du pain que quelqu'un qui ne sait pas - à condition d'ailleurs qu'il le sache concrètement, dans l'action. » (Perraud, 2018b).

7. Les activités professionnelles des ESAT se pratiquent sous la forme d'ateliers. Des travailleurs y sont encadrés par des moniteurs d'atelier spécialistes de l'activité professionnelle en question. Notre étude a concerné également les cinq ateliers des espaces verts (activité d'horticulture) et l'atelier de la serre (activité de production et de vente floricole et maraîchère).

8. Nous nous appuyons sur l'idée suivante: «De manière habituelle, l'apprentissage d'une connaissance se réalise dans un mouvement qui va du concret vers l'abstrait, de la catégorisation des objets vers leur désignation symbolique. Une formule est alors considérée comme un moyen d'appréhender une idée. Mais cette idée peut être appréhendée différemment par tout un chacun, suivant la réalité à laquelle il se réfère. Pour donner sens à une formule abstraite et pour que son sens soit partagé au sein d'une institution, il est pertinent de concrétiser une idée, de lui donner une forme de vie et de langage dans une situation précise. Une telle «ascension de l'abstrait au concret » peut être alors une modalité de transmission des savoirs au sein de cette institution. » (CDpE, 2019).

9. Dans l'atelier restauration, tous les jeudis à $13 \mathrm{~h} 30$ pendant toute la durée du projet.

10. Journal de terrain du 15 juin au 19 juin 2015.

11. Extrait de la transcription de la troisième réunion de l'ingénierie coopérative, IC3.

12. Pour des raisons de place, nous avons recouru à la forme acronymique «APC " pour signifier l'arrière-plan commun sur le schéma.

13. Le glossaire de l'ouvrage Didactique pour enseigner, propose la définition suivante : « la TACD reprend la notion, développée par Ludwig Wittgenstein, de jeu de langage-forme de vie pour souligner que tout énoncé, tout élément linguistique, pour être compris, doit être référé au système linguistique dans lequel il fonctionne, qu'on peut considérer comme une sorte de jargon fait de termes, d'expressions, de questions-réponses au sein d'un dialogue spécifique, etc. Ce jargon est un jeu de langage. Ce jeu de langage doit être lui-même situé dans la pratique, dans l'usage, qui lui donne son sens. Cette pratique qui détermine le sens des énoncés, c'est la forme de vie » (CDpE, 2019).

14. «Jack goody et l'empire de la littératie - chapitre 1 », Une ressource pédagogique en anthropologie de l'écrit, proposée par Jean-Marie Privat et Mohammed Kara, coproduite par l'Université de Lorraine et l'Université Ouverte des Humanités. 


\section{RÉSUMÉS}

La question de la place accordée aux savoirs fondamentaux (Lire, écrire et compter), pour des adultes en situation de handicap intellectuel, reste une question vive dans notre société où l'écrit est omniprésent. Dans cet article, nous allons décrire et analyser les premiers pas d'une coopération entre des acteurs d'un collectif, engagés dans une ingénierie coopérative (Sensevy, 2011), dont les champs de pratique sont a priori éloignés: des professionnels d'un ESAT (établissement service d'aide par le travail), des adultes en situation de handicap (appelés travailleurs) et une chercheure (l'auteure de cet article. Plus précisément, nous répondrons aux questions suivantes : que se passe-t-il quand des acteurs de cultures différentes apprennent à travailler ensemble? Qu'est-ce que cette coopération inédite transforme? Pour ce faire, nous nous centrerons sur la coopération dans l'atelier restauration de l'ESAT, entre un travailleur, Walter, les monitrices de cet atelier et la chercheure. Ensemble, ils vont élaborer un outil d'accessibilité, appelé cartes-constellations, permettant à Walter de mobiliser le comptage dans son activité professionnelle.

For intellectually disabled adults, the place of fundamental knowledge (reading, writing and counting) remains a live question in our society where writing is omnipresent. This article is a description and an analysis of the first steps of an cooperation between members of a collective committed in a cooperative engineering (Sensevy, 2011) of which practice fields seem at first view distant from one another: staff in an ESAT (Etablissement Service d'Aide par le Travail: institution for help to disabled adults through work) adults with disabilities (called workers) and a researcher (the author of this article). More specifically, we will answer the following questions: what happens when actors from different cultures learn to work together? What is this new cooperation transforming? To do this, we will focus on cooperation in the ESAT restoration workshop between a worker, Walter, the instructors of this workshop and the researcher. Together, they will develop an accessibility tool, called constellation cards, that will allow Walter to mobilize counting in his professional activity.

\section{INDEX}

Keywords : cooperative engineering, thought collective, JATD (Joint action theory in didactics), intellectual disability, ESAT (institution for help to disabled adults through work)

Mots-clés : ingénierie coopérative, collectif de pensée, TACD, handicap intellectuel, ESAT

\section{AUTEUR}

\section{CAROLINE PERRAUD}

Docteure en sciences de l'éducation, membre associée du laboratoire CREAD, EA 3875, \& chargée de mission à l'Apei ouest 44, association membre de l'UNAPEI 